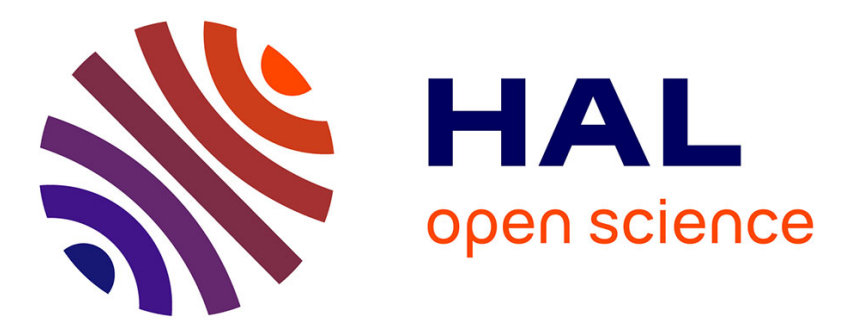

\title{
Nanoselective area growth and characterization of dislocation-free InGaN nanopyramids on AlN buffered $\mathrm{Si}(111)$ templates
}

Suresh Sundaram, Youssef El Gmili, Renaud Puybaret, Li X., P.L. Bonanno, Konstantinos Pantzas, Gilles Patriarche, Paul L. Voss, Jean-Paul Salvestrini, Abdallah Ougazzaden

\section{To cite this version:}

Suresh Sundaram, Youssef El Gmili, Renaud Puybaret, Li X., P.L. Bonanno, et al.. Nanoselective area growth and characterization of dislocation-free InGaN nanopyramids on AlN buffered $\mathrm{Si}(111)$ templates. Applied Physics Letters, 2015, 107 (113105), 10.1063/1.4931132 . hal-01203766

\section{HAL Id: hal-01203766 https://hal.science/hal-01203766}

Submitted on 1 Feb 2022

HAL is a multi-disciplinary open access archive for the deposit and dissemination of scientific research documents, whether they are published or not. The documents may come from teaching and research institutions in France or abroad, or from public or private research centers.
L'archive ouverte pluridisciplinaire HAL, est destinée au dépôt et à la diffusion de documents scientifiques de niveau recherche, publiés ou non, émanant des établissements d'enseignement et de recherche français ou étrangers, des laboratoires publics ou privés. 


\title{
Nanoselective area growth and characterization of dislocation-free InGaN nanopyramids on AIN buffered Si(111) templates
}

\author{
S. Sundaram, ${ }^{1}$ Y. El Gmili, ${ }^{1}$ R. Puybaret, ${ }^{1,2}$ X. Li, ${ }^{1,2}$ P. L. Bonanno, ${ }^{1,2}$ K. Pantzas ${ }^{3}$ \\ G. Patriarche, ${ }^{3}$ P. L. Voss, ${ }^{1,2}$ J. P. Salvestrini, ${ }^{4}$ and A. Ougazzaden ${ }^{1,2, a)}$ \\ ${ }^{1}$ CNRS, UMI 2958 Georgia Tech-CNRS, 2 Rue Marconi, 57070 Metz, France \\ ${ }^{2}$ School of Electrical and Computer Engineering, Georgia Institute of Technology, GT Lorraine, \\ 57070 Metz, France \\ ${ }^{3}$ CNRS, UPR LPN, Route de Nozay, 91460 Marcoussis, France \\ ${ }^{4}$ Universite de Lorraine, Centrale Supelec, LMOPS, EA 4423, 2 rue E. Belin, 57070 Metz, France
}

(Received 27 March 2015; accepted 6 September 2015; published online 16 September 2015)

\begin{abstract}
We report the metal organic chemical vapor deposition growth of dislocation-free $100 \mathrm{~nm}$ thick hexagonal $\mathrm{InGaN}$ nanopyramid arrays with up to $33 \%$ of indium content by nano-selective area growth on patterned AlN/Si (111) substrates. InGaN grown on $\mathrm{SiO}_{2}$ patterned templates exhibit high selectivity. Their single crystal structure is confirmed by scanning transmission electron microscope combined with an energy dispersive X-ray analysis, which also reveals the absence of threading dislocations in the InGaN nanopyramids due to elastic strain relaxation mechanisms. Cathodoluminescence measurements on a single InGaN nanopyramid clearly show an improvement of the optical properties when compared to planar InGaN grown under the same conditions. The good structural, morphological, and optical quality of the InGaN nanostructures grown on $\mathrm{AlN} / \mathrm{Si}$ indicates that the nano-selective area growth technology is attractive for the realization of site-controlled indium-rich InGaN nanostructure-based devices and can also be transferred to other highly mismatched substrates. (C) 2015 AIP Publishing LLC. [http://dx.doi.org/10.1063/1.4931132]
\end{abstract}

The band gap of InGaN alloys can be tuned across the whole visible spectrum and into the infrared, making InGaN a strong candidate for a wide range of optoelectronic ${ }^{1}$ and photovoltaic devices. ${ }^{2}$ Even though multiple quantum well ${ }^{3,4}$ or multi-layered ${ }^{5}$ architectures have been used to delay strain relaxation, the growth of thick, high quality, InGaN layers (beyond $15 \%$ of In incorporation) even on GaN templates remains challenging. ${ }^{4,6}$ Lattice mismatch induced strain relaxation, phase separation, and transition from 2-D to 3-D growth result in $\mathrm{InGaN}$ epilayers with a large density of defects and In composition inhomogeneity. These detrimental phenomena are even more pronounced in the case of $2 \mathrm{D}$ InGaN layers grown on silicon, resulting in low crystalline quality and large compositional and morphological nonuniformity, as evidenced by several authors who studied InGaN layers (with up to $40 \%$ of In content) on AlN-buffered silicon (111) substrates. ${ }^{7-9}$ Thus, it is desirable to develop approaches that can improve the quality of thick InGaN structures with high indium content on AlN/Si templates.

Luryi and Suhir ${ }^{10}$ theoretically described the heteroepitaxial growth on small seed pads with nanoscale lateral dimension which allows the accommodation of stress without dislocation generation. Adopting this approach can help to mitigate the intrinsic InGaN stress related problems and substantially improve material quality and subsequent device performances. Significant results have been reported for selfassembled InGaN nanostructures over the entire composition range using hydride vapour phase epitaxy. However, this process leads to random distribution of island sizes. ${ }^{11}$ Albert et al. reported selective area MBE growth of InGaN

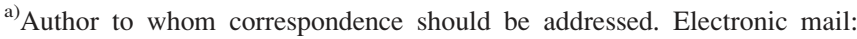
abdallah.ougazzaden@georgiatech-metz.fr.
}

nanostructures on GaN nanocolumns on buffered silicon substrates emitting from the ultraviolet $(3.2 \mathrm{eV})$ to infrared $(0.78 \mathrm{eV}) .{ }^{12}$ Further studies show that it is difficult to grow high quality In rich InGaN nanostructures on GaN nanocolumns without interfacial defects. ${ }^{13}$ Using metal organic chemical vapor deposition (MOCVD), which is used for industrial production of Light Emitting Diodes (LEDs), both $\mathrm{GaN}$ and InGaN nanostructures have been obtained on GaN templates. Good quality, InGaN nanostructures with around $20 \%$ In incorporation have been selectively grown on the GaN templates by either pulsing the precursors, ${ }^{14-16}$ or using the continuous precursors flow method. ${ }^{17,18}$ On silicon substrates, GaN Nano-Selective Area Growth (NSAG) has been demonstrated previously by MOCVD. ${ }^{19}$ A recent study has found temperature and pressure conditions for optimal NSAG InGaN growth on $200 \mathrm{~nm}$ openings by MOCVD. ${ }^{20}$ For opening sizes less than $100 \mathrm{~nm}$, the growth dynamics are expected to change, resulting mostly in single-crystal, defectfree nanostructures. ${ }^{21}$ We report on MOCVD growth of InGaN nanopyramids with up to $33 \%$ indium content on $80 \mathrm{~nm}$ mask openings. Extensive characterization by transmission electron microscopy (TEM) and energy dispersive X-ray analysis (EDX) confirms that these NSAG conditions result in singlecrystal, dislocation-free nanostructures, and allow measurement of indium content at different parts of the nanostructures. Cathodoluminescence (CL), scanning electron microscopy (SEM), and X-ray diffraction (XRD) measurements provide additional insight.

Our process begins with silicon (111) substrates, which were pre-coated with 200-nm nanocrystalline AlN by physical vapor deposition. Then a $100 \mathrm{~nm}$ thick negative-tone resist (hydrogen silsesquioxane) was spin-coated and patterned using electron-beam lithography, leaving a hydrogenated $\mathrm{SiO}_{2}$ 

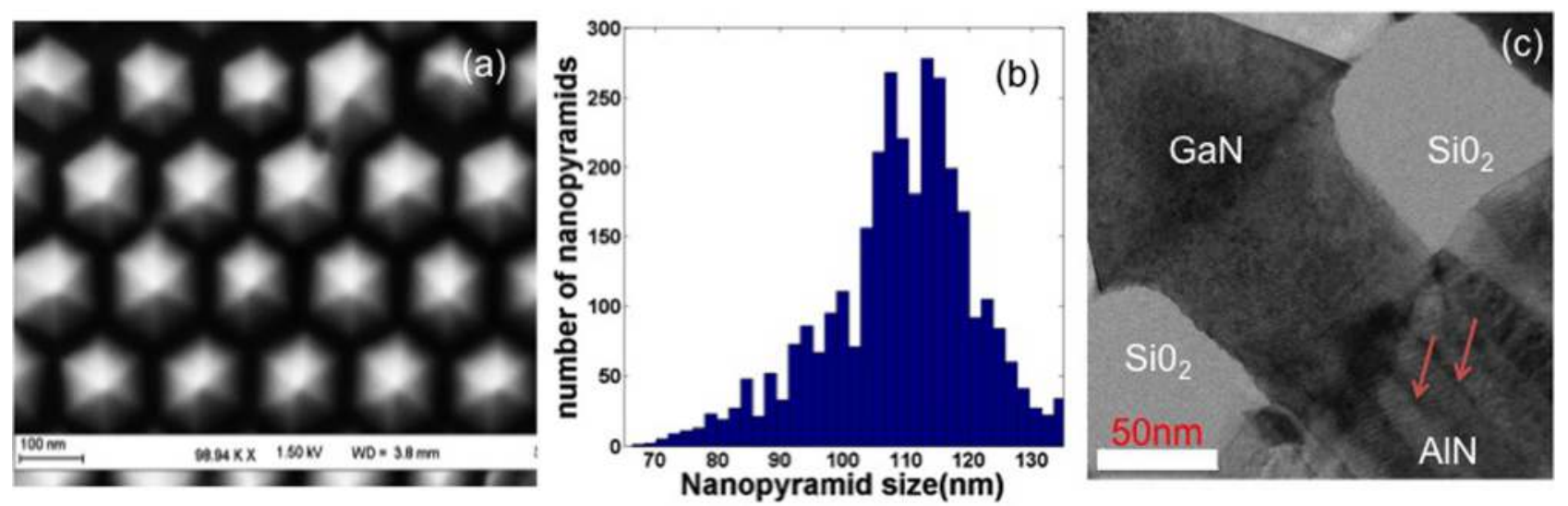

FIG. 1. (a) SEM image of the GaN nanopyramids grown on circular openings on the patterned AlN/Si substrate. (b) Size (distance between the two opposite edges) distribution histogram of the GaN nanopyramids extracted from the SEM images. (c) Cross sectional STEM images of a single GaN nanopyramid grown on patterned AlN/Si templates.

mask. A tetramethyl ammonium hydroxide etch was then used to open nano-holes with diameter of around $80 \mathrm{~nm}$. A full description of the mask patterning process can be found elsewhere. ${ }^{17}$ The growth of GaN nanostructures was carried out first to optimize the growth process independent of indium content, and then InGaN nanostructures were grown separately on the patterned templates using a MOCVD reactor. ${ }^{22}$ Trimethylgallium (TMGa), trimethylindium, and ammonia were used as growth precursors under nitrogen ambient. In the case of $\mathrm{InGaN}$ growth, a $20 \mathrm{~nm}$ thick $\mathrm{GaN}$ layer was first grown on the AlN buffer layer. The growth temperatures measured at the susceptor below the wafer were $1000^{\circ} \mathrm{C}$ and $780^{\circ} \mathrm{C}$ for $\mathrm{GaN}$ and $\mathrm{InGaN}$, respectively. The structural and morphological properties of the nanostructures were studied using high resolution XRD (HR-XRD), SEM, and scanning TEM (STEM) with EDX. The optical properties of the nanostructures at $77 \mathrm{~K}$ were studied using depth-resolved CL.

Fig. 1(a) shows a SEM image of the GaN nanopyramids grown on circular openings on the patterned AlN/Si substrate. High growth selectivity without any polycrystalline deposits on the masks is obtained.

Fig. 1(a) also clearly shows that each nanopyramid exhibits six smooth triangular sidewall semipolar facets corresponding to the $\left\{\begin{array}{llll}1 & -1 & 0 & 1\end{array}\right\}$ family of planes. More than $99.6 \%$ of the nanopyramids are hexagonal and have six clearly identifiable triangular facets. Fig. 1(b) shows the size distribution histogram of more than 3000 nanopyramids, derived from SEM image analysis. The average size of the GaN nanopyramids, defined by the distance between the two opposite edges, is found to be $110 \mathrm{~nm}$. More than $90 \%$ of the nanopyramids have sizes in the $110 \pm 20 \mathrm{~nm}$ range. The excellent morphological quality of the nanopyramids may result from the fact that no plasmas or reactive ion bombardment are used in the mask processing, preventing damage to nucleation sites. Fig. 1(c) shows a bright field STEM image of a single GaN nanopyramid grown on the AlN/Si template. It clearly shows that the 200-nm thick AlN buffer consists of polycrystalline nanocolumns. The dark veins (indicated by red arrows) correspond to grain boundaries. The scanning TEM image also shows most of a $200 \mathrm{~nm}$ high GaN monocrystalline nanopyramid. The nanopyramid is a single crystal because the small $80-\mathrm{nm}$ diameter mask opening lowers the nucleation rate, so the first nucleation on the AIN seed is the only nucleation on AlN. ${ }^{21}$ The growth dynamics should result in full strain relaxation due to the availability of lateral free surfaces that allow the accommodation of stress without dislocation generation.

After the optimization of the GaN nano-heteroepitaxy, InGaN nanostructures were grown on similarly patterned AlN/Si templates. For comparison, the structural and morphological characteristics of planar InGaN (outside of the patterned area: unmasked area) have been studied. Fig. 2 shows the corresponding HR-XRD $2 \boldsymbol{\theta}-\boldsymbol{\omega}$ scan with its simulated fit for the (002) reflection planes. The right inset of Fig. 2 shows the reciprocal space map (RSM) for the InGaN asymmetric (114) peak and an SEM image of the InGaN surface. The HR-XRD 20- $\boldsymbol{\omega}$ scan shows three distinct peaks that can be attributed to (002) reflections of the AlN buffer layer, GaN layer, and InGaN layer. XRD simulation gives an average of $28 \%$ indium incorporation in the planar InGaN layer. The RSM of the control sample (Fig. 2, right inset) reveals a bright InGaN diffraction spot and indicates that the InGaN layer is fully relaxed on the AlN seed layer. As expected, the planar InGaN exhibits 3D growth with V-pits and trench defects resulting in the rough morphological quality evidenced by the SEM image in the left inset of Fig. 2.

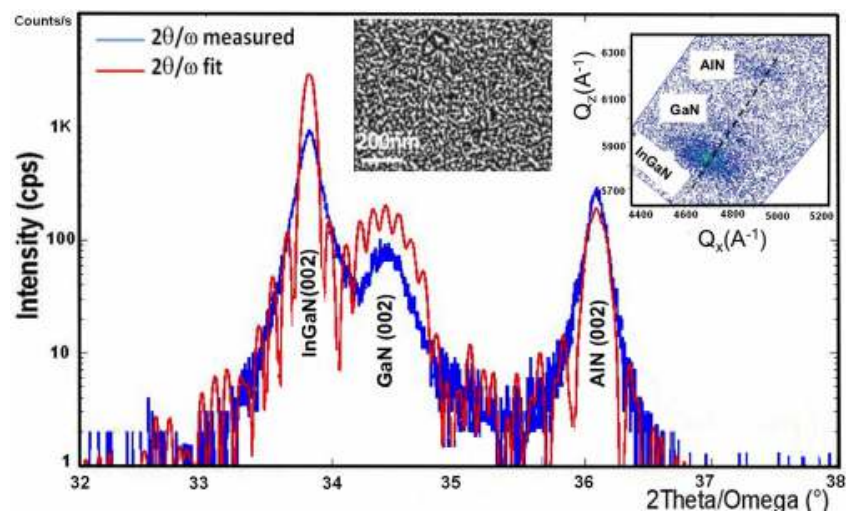

FIG. 2. HR-XRD $2 \theta-\omega$ scan with its simulated fit for the (002) reflection of planar InGaN (outside of the patterned area). Insets show the SEM image of planar InGaN (left inset) and the RSM (right inset). 

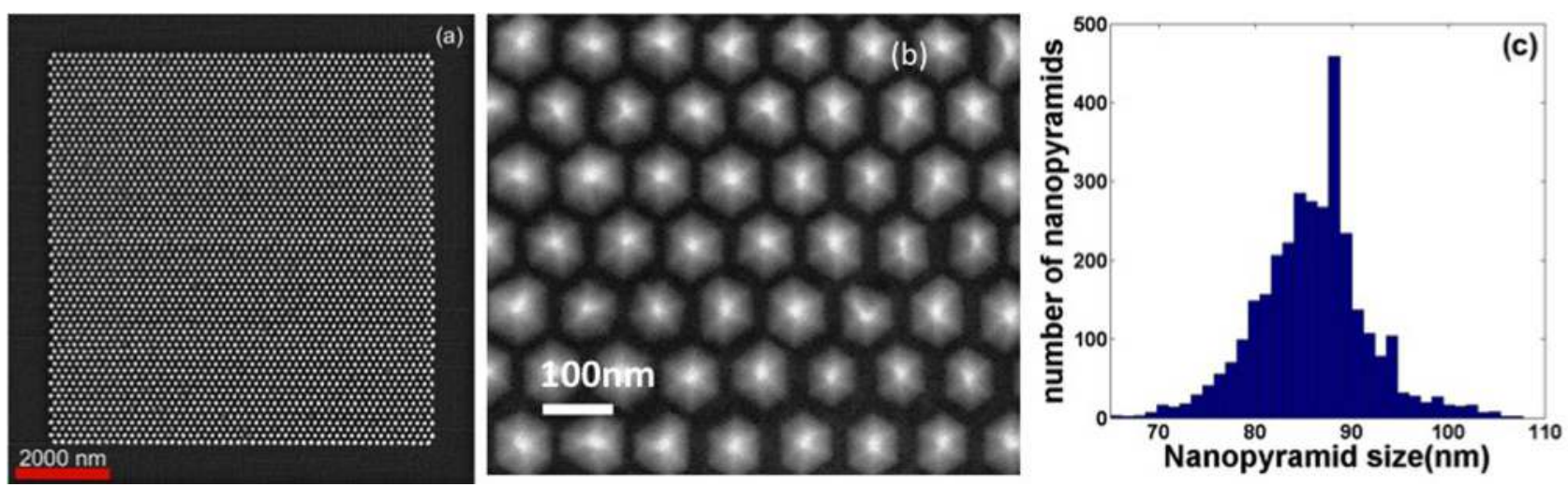

FIG. 3. (a) SEM image of the InGaN nanopyramids grown on the patterned AlN/Si template. The size (distance between the two opposite edges) of the patterned area is $10 \times 10 \mu \mathrm{m}^{2}$. (b) Higher magnification SEM image of the InGaN nanopyramids. (c) Size distribution histogram of InGaN nanopyramids grown on $\mathrm{AlN} / \mathrm{Si}(111)$.

Figs. 3(a) and 3(b) show the SEM images, obtained at different magnifications, of InGaN nanostructures grown on the circular openings of the patterned AlN/Si template. Similar to the GaN nanopyramids grown on AlN/Si (see Fig. 1(a)), InGaN nanopyramids also clearly show six smooth sidewall semipolar facets with perfect selectivity throughout the whole $10 \times 10 \mu \mathrm{m}^{2}$ patterned area without any polycrystalline deposits. These results are similar to those obtained in the growth of InGaN nanopyramids on $\mathrm{GaN}$ templates. ${ }^{15}$ The statistical analysis of the SEM image of Fig. 3(a) shows that $91 \%$ of the InGaN nanopyramids grown on the $\mathrm{AlN} / \mathrm{Si}$ template have a good hexagonal shape with clear facets. It should be noted that both $\mathrm{GaN}$ and $\mathrm{InGaN}$ nanopyramids grown on AIN/Si templates have almost the same shape uniformity. From the size (distance between the two opposite edges) distribution histogram of $\mathrm{InGaN}$ nanopyramids grown on AlN/Si (111) shown in Fig. 3(c), we can derive the average size of the InGaN nanopyramids, which is found to be equal to $86 \mathrm{~nm}$. More than $90 \%$ of the nanopyramids have sizes in the $86 \pm 7 \mathrm{~nm}$ range.

To investigate the structural quality and composition of the InGaN nanopyramids, cross-sectional STEM studies were carried out. Figs. 4(a) and 4(b) show bright field STEM images of both planar $\mathrm{InGaN}$ and $\mathrm{InGaN}$ nanopyramids
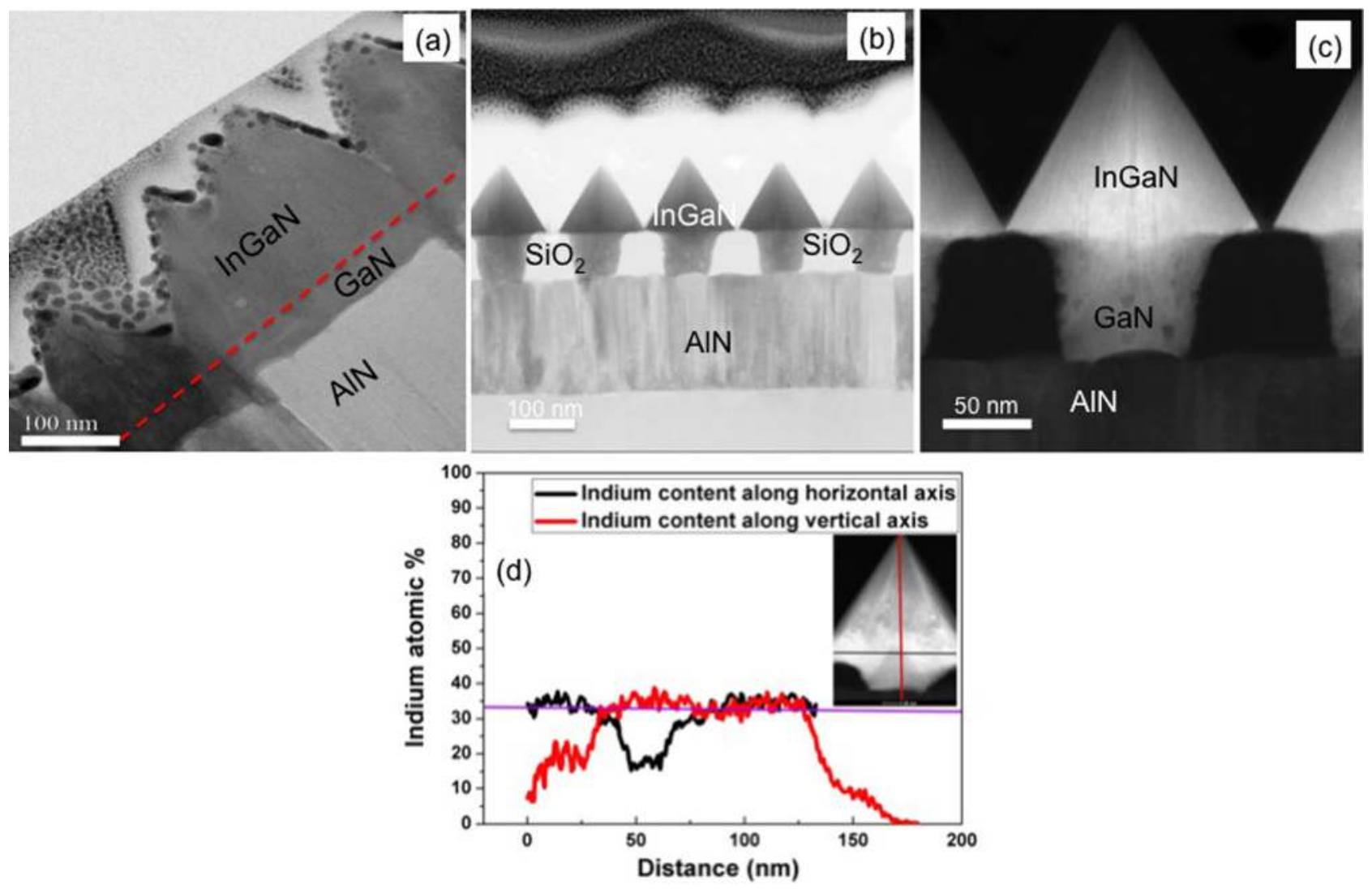

FIG. 4. (a) Cross sectional BF-STEM image of planar InGaN grown on the non-patterned part of the AlN/Si template. (b) Cross sectional BF-STEM image of InGaN nanopyramid arrays grown on the patterned AlN/Si template. (c) Cross sectional HAADF-STEM images of single InGaN nanopyramid. (d) EDX line scans for indium in InGaN nanopyramids along the vertical (red) and horizontal (black) axes of the nanopyramid. 
grown on AlN/Si. The planar InGaN surface is completely 3D with networks of $\mathrm{V}$-pits originating from the threading dislocations propagating from the nanocrystalline AlN buffer layer. In contrast, the InGaN nanopyramids on patterned AlN/Si templates are monocrystalline and dislocation free with very clear interfaces between $\mathrm{InGaN} / \mathrm{GaN}$ and nanocrystalline AlN as shown in the High Angle Annular Dark Field Scanning Transmission Electron Microscope (HAADF STEM) image in Fig. 4(c). This shows that the nanopyramids start from a single nucleus on the AlN seed because of the small opening, and the strain relaxation is purely elastic without generation of further defects and misfit dislocations, implying that nano-selective area growth has mitigated the strain-induced degradation that is present in the InGaN 2D epilayers (shown in the left inset of Fig. 2), which indicates the absence of large scale indium composition inhomogeneity, i.e., indium clustering and composition pulling. This single crystal result is consistent with our GaN on AlN/Si results and other NSAG results reported by Ikejiri et al. ${ }^{21}$ The vertical and horizontal EDX line scan on the nanopyramid for indium atomic concentrations also confirms this, which is shown in Fig. 4(d). It clearly shows that the nanopyramids have uniform indium incorporation averaging around $33 \%$, demonstrating the ability of NSAG to obtain very uniform and homogeneous InGaN nanostructures without any misfit defects. We note that cross-sectional EDX is less accurate at the apex of the nanopyramids. This is a wellknown characteristic of EDX near interfaces.

CL spectroscopy was used to study the emission characteristics of a single InGaN nanopyramid. Fig. 5 shows low temperature $(77 \mathrm{~K}) \mathrm{CL}$ emission spectra from a single $\mathrm{InGaN}$ nanopyramid and the inset shows the CL spectra from planar InGaN in the field (unmasked area). At these low beam energies $(5-7 \mathrm{keV})$, the electron beam energy corresponds to a depth of maximum energy loss varying in the range of $40-60 \mathrm{~nm}$, and thus, the spectra consist of luminescence from the $100 \mathrm{~nm}$ thick InGaN region. Planar InGaN exhibits two luminescence bands, which are centered at $562 \mathrm{~nm}$ and $700 \mathrm{~nm}$. The short wavelength peak can be attributed to the near band edge emission of InGaN with an In incorporation

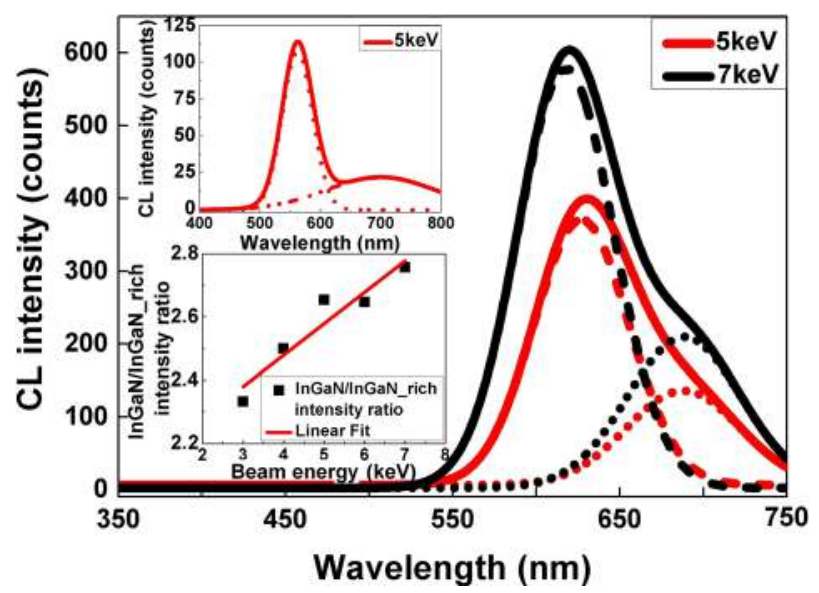

FIG. 5. Low temperature ( $77 \mathrm{~K}$ ) CL emission spectra from a single InGaN nanopyramid with respective peak deconvolutions in dashed lines. Top inset shows the CL spectra and peak deconvolutions from the InGaN field and the bottom inset shows the variation of peak intensity and its ratio with energy beam. equal to $25 \%$ (In composition is determined according to the work of Orsal et al. ${ }^{23}$ taking into account both the relaxation rate of the layer and the associated bandgap bowing parameter). The other luminescence band spanning from $550 \mathrm{~nm}$ to $750 \mathrm{~nm}$ originates probably from the localization of excitons at potential minima in In-rich $\mathrm{InGaN}$ areas confirming the expected In content fluctuations in planar InGaN. In the nanopyramid, a large luminescence band centered at $625 \mathrm{~nm}$ corresponding to In incorporation of $30 \%$ can be observed. A second weaker luminescence band centered at $680 \mathrm{~nm}$ (In incorporation of $33 \%$ ) can also be seen. Thus, under the same growth conditions, higher In incorporation in the nanopyramids than in InGaN field is obtained. Under similar excitation $(5 \mathrm{keV})$, comparing the largest luminescence bands, almost four times larger peak intensity is obtained from the InGaN nanopyramids than from planar InGaN, likely due to higher crystalline quality in the nanopyramid.

In the nanopyramid, with the increasing of the electron beam energy, the ratio between the low ( $\mathrm{InGaN}$ ) and high (InGaN-rich) wavelength peak intensities increases monotonically as shown in the bottom inset of Fig. 5, indicating that the In incorporation in that nanopyramid is slightly larger in the top $(33 \%)$ than in the base $(30 \%)$ of the nanopyramid.

In conclusion, we have demonstrated nano-selective area growth of $\mathrm{GaN}$ and $\mathrm{InGaN}$ nanopyramids on $\mathrm{AlN} /$ Si(111) using MOCVD. This approach yields perfectly selective single crystal nanopyramids on silicon substrates. The nanopyramids are dislocation free. Homogeneously distributed InGaN nanopyramids with $\sim 33 \%$ In incorporation have been obtained. Luminescence emission analysis confirms the high optical quality and enhancement of indium incorporation in the nanostructures. This proof-of-concept result may help to overcome current limitations in the growth of high quality thick InGaN nanostructured devices on low-cost substrates by MOCVD. Although this paper presents the results of NSAG growth of high quality GaN and InGaN nanostructures on nanocrystalline AlN on silicon, the method that we present is generic and can be used for deposition on any inexpensive, III-N compatible substrate. This makes the process attractive as a batch process for industrial applications.

This work was supported by the French National Research Agency (ANR), under the GANEX LABEX Program (ANR11-LABX-0014), NOVAGAINS Project (ANR-12-PRGE0014-02), and CNRS INCEPT PEPS project. The authors would like to thank C. Pradalier for the numerical analysis of the SEM images and D. Troadec for FIB TEM preparation.

${ }^{1}$ S. Nakamura and G. Fasol, The Blue Laser Diode (Springer, Berlin, 1997). ${ }^{2}$ O. Jani, I. Ferguson, C. Honsberg, and S. Kurtz, Appl. Phys. Lett. 91, 132117 (2007).

${ }^{3}$ N. G. Young, R. M. Farrell, Y. L. Hu, Y. Terao, M. Iza, S. Keller, S. P. DenBaars, S. Nakamura, and J. S. Speck, Appl. Phys. Lett. 103, 173903 (2013).

${ }^{4}$ L. Redaelli, A. Mukhtarova, S. Valdueza-Felip, A. Ajay, C. Bougerol, C. Himwas, J. Faure-Vincent, C. Durand, J. Eymery, and E. Monroy, Appl. Phys. Lett. 105, 131105 (2014).

${ }^{5}$ Y. El Gmili, G. Orsal, K. Pantzas, T. Moudakir, S. Sundaram, G. Patriarche, J. Hester, A. Ahaitouf, J. P. Salvestrini, and A. Ougazzaden, Acta Mater. 61, 6587 (2013).

${ }^{6}$ Y. El Gmili, G. Orsal, K. Pantzas, A. Ahaitouf, T. Moudakir, S. Gautier, G. Patriarche, D. Troadec, J. P. Salvestrini, and A. Ougazzaden, Opt. Mater. Express 3, 1111 (2013). 
${ }^{7}$ A. G. Bhuiyan, A. Mihara, T. Esaki, K. Sugita, A. Hashimoto, A. Yamamoto, N. Watan-abe, H. Yokoyama, and N. Shigekawa, Phys. Status Solidi 9, 670 (2012).

${ }^{8}$ I. Gherasoiu, K. M. Yu, L. A. Reichertz, V. M. Kao, M. Hawkridge, J. W. Ager, and W. Walukiewicz, Phys. Status Solidi 247, 1747 (2010).

${ }^{9}$ A. Yamamoto, A. Mihara, Y. Zheng, and N. Shigekawa, Jpn. J. Appl. Phys., Part 1 52, 08JB19 (2013).

${ }^{10}$ S. Luryi and E. Suhir, Appl. Phys. Lett. 49, 140 (1986).

${ }^{11}$ T. Kuykendall, P. Ulrich, S. Aloni, and P. Yang, Nature Mater. 6, 951 (2007).

${ }^{12}$ S. Albert, A. Bengoechea-Encabo, M. A. Sanchez-Garca, X. Kong, A. Trampert, and E. Calleja, Nanotechnology 24, 175303 (2013).

${ }^{13}$ X. Kong, S. Albert, A. Bengoechea-Encabo, M. A. Sanchez-Garcia, E. Calleja, and A. Trampert, Phys. Status Solidi A 212, 736 (2015).

${ }^{14}$ Y. Wang, K. Zang, S. Chua, M. S. Sander, S. Tripathy, and G. Fonstad, J. Phys. Chem. B 110, 11081 (2006).

${ }^{15}$ V. Jindal, N. Tripathi, M. Tungare, O. Paschos, P. Haldar, and F. Shahedipour-Sandvik, Phys. Status Solidi 5, 1709 (2008).
${ }^{16}$ J. Song, B. Leung, Y. Zhang, and J. Han, Nanotechnology 25, 225602 (2014).

${ }^{17}$ S. Sundaram, R. Puybaret, Y. El Gmili, X. Li, P. L. Bonanno, K. Pantzas, G. Orsal, D. Troadec, Z. H. Cai, G. Patriarche, P. L. Voss, J. P. Salvestrini, and A. Ougazzaden, J. Appl. Phys. 116, 163105 (2014).

${ }^{18}$ S. Sundaram, R. Puybaret, X. Li, Y. El Gmili, J. Streque, G. Orsal, G. Patriarche, P. L. Voss, J. P. Salvestrini, and A. Ougazzaden, Phys. Status Solidi A 212, 740 (2015).

${ }^{19}$ D. Zubia, S. H. Zaidi, S. R. J. Brueck, and S. D. Hersee, Appl. Phys. Lett. 76, 858 (2000).

${ }^{20}$ J. W. Ho, R. J. N. Tan, M. Heuken, A. A. O. Tay, and S. J. Chua, J. Cryst. Growth 420, 64 (2015).

${ }^{21}$ K. Ikejiri, F. Ishizaka, K. Tomioka, and T. Fukui, Nanotechnology 24, 115304 (2013).

${ }^{22}$ S. Gautier, C. Sartel, S. Ould-Saad, J. Martin, A. Sirenko, and A. Ougazzaden, J. Cryst. Growth 298, 428 (2007).

${ }^{23}$ G. Orsal, Y. El Gmili, N. Fressengeas, J. Streque, R. Djerboub, T. Moudakir, S. Sundaram, A. Ougazzaden, and J. P. Salvestrini, Opt. Mater. Express 4, 1030 (2014). 